\title{
The ATLAS RPC detector as a Luminosity monitor
}

\author{
Marcello Bindi*t \\ University and INFN of Bologna \\ E-mail: Marcello.Bindiecern.ch
}

\begin{abstract}
The ATLAS RPC Detector Control System has been mainly used to monitor and safely operate the RPC detector during 2010 and 2011 LHC run. The large number ( 3600) of gap currents, individually monitored with $\mathrm{nA}$ accuracy, allowed us to study the detector behaviour with growing luminosity and beam currents. A clear linear correlation between the average gap current and the luminosity has been observed over more than four orders of magnitude. This publication will demonstrate how this method has a good potential for improving the accuracy and the stability of the standard luminosity measurements on a long time basis.
\end{abstract}

XI workshop on Resistive Plate Chambers and Related Detectors - RCP2012,

February 5-10, 2012

INFN Laboratori Nazionali di Frascati Italy

\footnotetext{
* Speaker.

${ }^{\dagger}$ on behalf of ATLAS Collaboration.
} 


\section{Introduction}

The Resistive Plate Chambers (RPCs)[1] of the ATLAS experiment[2, 3] have been providing an independent muon trigger and a two-coordinate measurement during the entire 2010-2011 data taking. As a result of a time resolution of the order of ns, the ATLAS RPCs were able to select muon trigger candidates and identify the relative bunch crossing with very high efficiency[4]. Furthermore, the possibility of time-of-flight studies has been recently explored[5].

The ATLAS RPCs cover a very broad surface of about $4000 \mathrm{~m}^{2}$; they are located in the muon barrel toroid and distributed in 3 concentric cylindrical layers that are respectively $5,7.5$ and $10 \mathrm{~m}$ away from the beam pipe. There are in total $\sim 3600$ gas gaps whose currents have been monitored by the RPCs Detector Control System (DCS) with nA accuracy [6]. The high granularity and quality of the current read out made it possible to exploit different detector characteristics. In particular, the stability of the currents was ensured by automatic adjustment of the high voltages working point.

All the conditions just mentioned seemed optimal to furnish an independent luminosity estimate as a cross check for the ATLAS experiment. The measurement is achieved by using DCS information, does not depend on the data acquisition process and consequently from the bunch structure. Furthermore, considering that the RPCs are few meters away from the beam pipe, the measurement is less sensitive to the beam halo background.

In this note we present the most relevant results, showing how it has been possible to find and quantify a clear relation between the RPC currents and the luminosity provided by the Large Hadron Collider (LHC) during the biennium 2010-2011. The goal is to provide ATLAS with an independent luminosity measurement for the 2012 data taking season.

A brief description of this measurement technique is presented in section 2. The first results together with an overview of the main systematic effects are shown is section 3, including an estimate of the contribution due to the cavern activation. A plan for a reliable luminosity measurement with ATLAS RPCs in the 2012 is presented in section 4, including a first comparison with the ATLAS luminosity official measurements. Finally some concluding remarks and the outlook are given in Section 5.

\section{Measurement technique}

A detailed description of the measurement technique can be found in references [7].

The RPC DCS is in primis responsible for a safe operation and monitoring of the detector. The gap currents of the single gas volumes are constantly monitored in order to spot any problematic behaviour related to bad gas supply or anomalous ageing of the chambers. Furthermore, after excluding the misbehaving gaps, they have been used to estimate the average current per surface unit ${ }^{1}$. This quantity, once corrected for the environmental variables and pedestal subtracted, appeared to be a good candidate for a luminosity measurement. Neverthless, to obtain a reliable relation between current and luminosity, a method of estimating the sudden current drop/increase associated with sudden changes in the beam status was used. This method allowed at the same time to minimize the systematics due to environmental factors.

\footnotetext{
${ }^{1}$ From now on we'll be always referring to the average current per surface unit when talking about RPCs current.
} 
a)

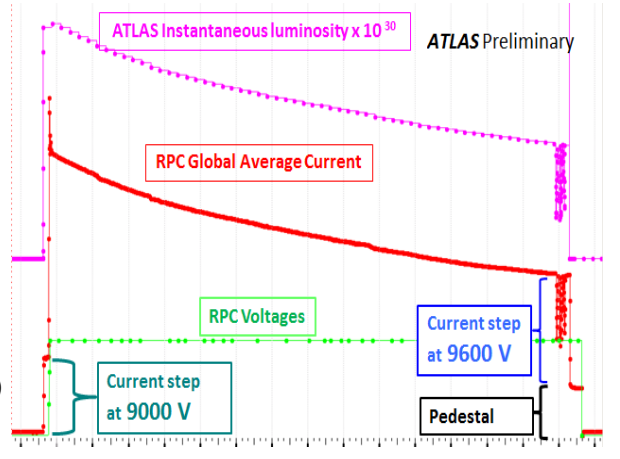
tioned was possible in the following cases: to STANDBY state.

The ATLAS RPCs were designed to automatically switch between two High Voltage (HV) configurations depending on the LHC beam conditions:

- the READY state, with the detector at full voltages/gain/efficiency $(9600 \mathrm{~V})$; the RPCs are required to reach that configuration as soon as the LHC declares stable beams;

- the STANDBY state, with the detector at lower voltages/gain/efficiency $(9000 \mathrm{~V})$; this configuration is requested whenever the LHC is injecting, ramping or squeezing the beams.

Taking into account the LHC operations, the use of the measurement method previously men-

- at the beginning of the fill, when the beams start colliding but before the stable beams declaration, in order to avoid the detector ramp up to READY state;

- at the end of the fill, when the beams are dumped and before the ramp down of the detector

The RPCs HV were at a fixed value during these beam transition phases which made it possible to estimate the contribution to the current due to the beam collisions. In the first case the detector was in STANDBY state; in the second case in READY state. The typical behaviour of the RPCs can

b)

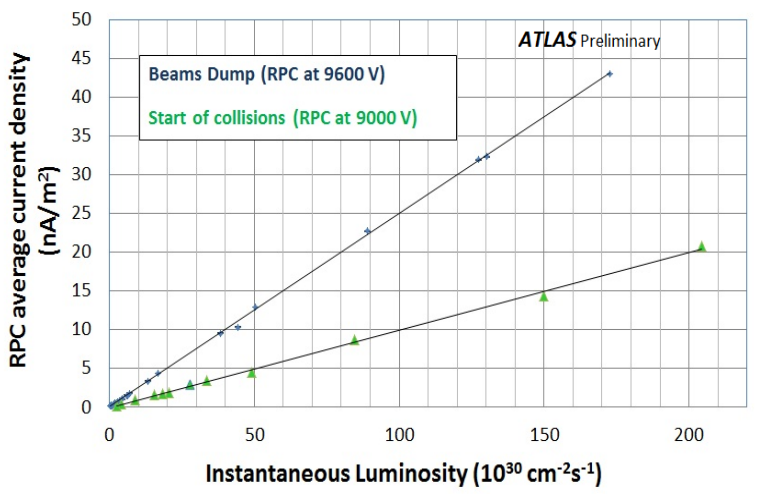

Figure 1: a )An example of the RPCs average current and the ATLAS inst. luminosity as a function of time before, during and after the LHC fill are shown. A pedestal is visible at the end of the fill, corresponding to the detector dark current. b ) The RPCs average current (per unit area and pedestal subtracted) versus the ATLAS inst. luminosity at the start of the collisions (green triangles) and at the beam dump (blue stars).

be observed by directly looking at a DCS plot (see Fig. 1 a), where the current follows the ATLAS instantaneous luminosity. A first positive step of the current can be seen at the beginning of the fill, when the detector was still at lower voltages $(9000 \mathrm{~V})$. At the end of the fill a negative step is also visible, occurring when the beams were dumped. Once the fill is over, a residual current (indicated as "Pedestal" in Fig. 1 a) is present, corresponding to the detector dark current at $9600 \mathrm{~V}$. The observed current difference $\Delta I$ is equal to the difference between the average detector current with beams and the average detector current without beams. This quantity was then associated to a luminosity difference $\Delta \mathscr{L}$.

Fig. $1 \mathrm{~b}$ shows the instantaneous luminosity versus the RPCs current for the two voltage gains. The data, obtained in several fills, show a clear linear relation between current and luminosity. The slopes of the two curves are different due to the different detector gains. 


\section{Results and main systematic effects}

One of the main systematic effects is the strong influence of the environmental parameters on the detector behaviour[4, 7]. A dynamic adjustment of the high voltage working point as a function of the local environmental conditions (temperature and atmospheric pressure) was necessary for a stable operation. For technical reasons it was only possible to apply this correction when the detector was at full voltage $(9600 \mathrm{~V})$. In this case the RPCs current was expected to be strictly correlated to the beam collisions and not influenced by other factors. Consequently the analysis efforts have been concentrated on the extraction of the corrected values of current at beam dumps.

Fig. 2 shows a collection of several measurements of average current density vs. instantaneous luminosity at beam dump. The measurements refer to 2010 and 2011 data and span over a range of more than 4 decades. Data fits a straight line with a slope of $0.312 \pm 0.001 \mathrm{nA} \mathrm{m} \mathrm{m}^{-2} / 10^{30} \mathrm{~cm}^{-2} \mathrm{~s}^{-1}$ and a negligible intercept. This result shows that the ratio between the ATLAS luminosity and the RPCs current $(\Delta I)$ is remarkably constant. Thus, demonstrating that an independent online luminosity measurement is possible once calibrated.

As a by product of this analysis, it was possible to extrapolate the fit to the LHC project luminosity of $\mathscr{L}=10^{34} \mathrm{~cm}^{-2} \mathrm{~s}^{-1}$. From the extracted value of the current, an average counting rate of $10 \mathrm{~Hz} / \mathrm{cm}^{-2}$ was predicted, by using a $30 \mathrm{pC} /$ count conversion factor. This result together with several 2-dimensional maps of the current have been extensively used for ATLAS upgrade studies, in order to have a reliable estimation and description of the hit rate for higher LHC luminosity. More details can be found on reference [7]

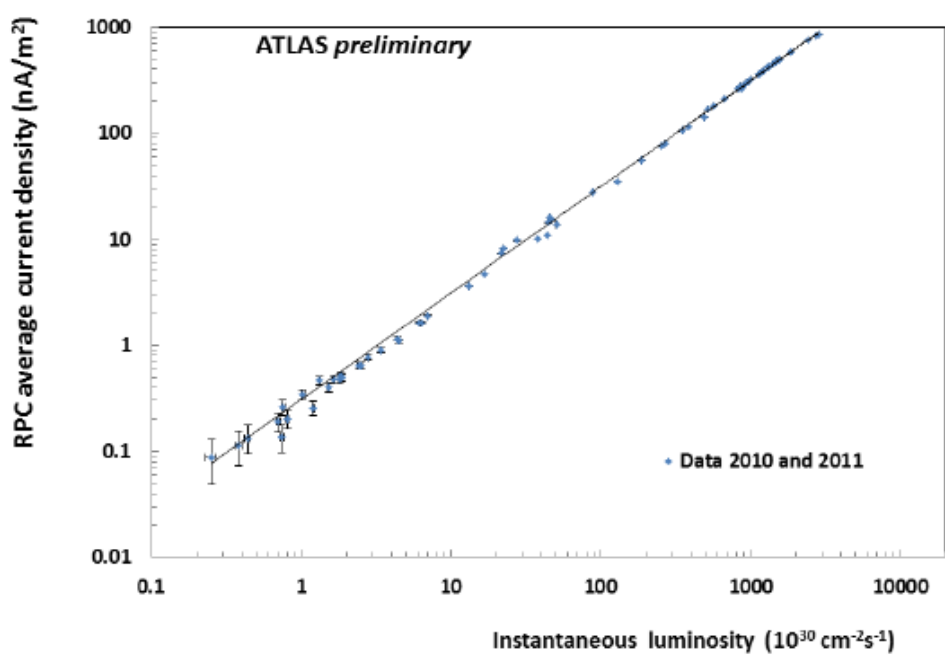

Figure 2: The RPC average current density as a function of the instantaneous luminosity at beam dump. The measurements include both 2010 and 2011 data and span over a range of more than four orders of magnitude. Data fit a straight line with a slope of $0.312 \pm 0.001 \mathrm{nA} \mathrm{m}^{-2} / 10^{30} \mathrm{~cm}^{-2} \mathrm{~s}^{-1}$ and a negligible intercept.

Looking in more detail at the current trends after beam dump, an interesting effect of ATLAS cavern activation has been observed. A very important effect that had to be understood, quantified and constantly monitored. This observation was possible even if the time needed by the DCS 
to update and calculate the average value of the currents was not optimal. Approximately every 30 seconds an independent data set was provided, thus implying a step in the RPCs current 3040 seconds after a beam dump. Rather than dropping to the expected detector dark current $(\sim$ $100 \mathrm{nA} \mathrm{m}^{-2}$ ), the trends were instead described by an exponential decay function.

The lifetime $(\tau)$ and the intensity $\left(A_{0}\right)$ of the exponential decay function have been extracted, analysing data for several fills with different instantaneous luminosities at beam dump. In order to have a reasonable amount of data to fit, the detector was kept regularly at full voltages for 20 minutes after each dump.

Figure 3 shows the results for a sub-sample of LHC fills where the RPCs current is shown as a function of time after beam dump for instantaneous luminosities ranging from 0.6 to 3.1 . $10^{33} \mathrm{~cm}^{-2} \mathrm{~s}^{-1}$.

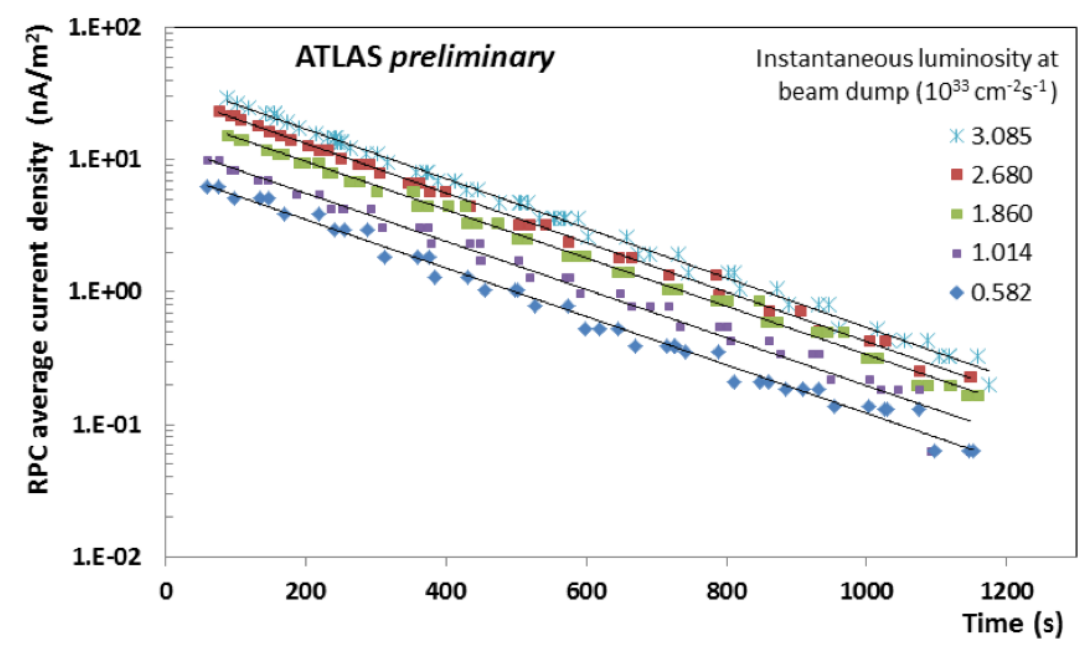

Figure 3: RPC average current density as a function of time after beam dump for different instantaneous luminosities.

The results of the fits are summarised in Table 1. The decay rate is almost independent from the instantaneous luminosity and has an estimated average lifetime of $\langle\tau\rangle=(234 \pm 1)$ s. This result is similar to the one found by the other ATLAS muon detectors $(\sim 200 \mathrm{~s})$; nevertheless its interpretation is still under study. The materials inside the RPCs, interested by the activation, are mainly Aluminium and Copper but the relative contribution of each element to the decay constant is not trivial. The value should be compared with $\tau \simeq 404 \mathrm{~s}$ for the $C u^{64}$, and $\tau \simeq 208 \mathrm{~s}$ for the Aluminium isotope $\mathrm{Al}_{13}^{28}$, formed if a neutron is added to the stable isotope $\mathrm{Al}_{13}^{27}$.

In Figure 4, the total current, the current induced by activation and their ratio (activation current/total current) are shown as a function of the instantaneous luminosity at beam dump. The activation current $\left(A_{0}\right)$ appears to depend linearly on the instantaneous luminosity. The ratio has been calculated and found to be almost constant with luminosity. The average value of the ratio is $(4.1 \pm 0.1) \%$.

\section{First comparison with ATLAS Luminosity measurements}

The ratio between the ATLAS luminosity and the current in the RPC is remarkably constant as 


\begin{tabular}{|c|c|c|c|c|}
\hline $\begin{array}{c}\text { Instantaneous Luminosity } \\
\left(\times 10^{33} \mathrm{~cm}^{-2} \mathrm{~s}^{-1}\right)\end{array}$ & $\mathbf{A}_{\mathbf{0}}\left(n A / m^{2}\right)$ & $\delta \mathbf{A}_{\mathbf{0}}\left(n A / m^{2}\right)$ & $\tau(s)$ & $\delta \tau(s)$ \\
\hline 3.085 & 40 & 1 & 232 & 2 \\
\hline 2.680 & 31 & 1 & 232 & 2 \\
\hline 1.860 & 22 & 1 & 235 & 3 \\
\hline 1.014 & 13 & 1 & 234 & 5 \\
\hline 0.582 & 8 & 1 & 237 & 4 \\
\hline
\end{tabular}

Table 1: The distributions have been fit with an exponential decay function $\mathrm{y}=\mathrm{A}_{0} * \exp (-\mathrm{t} / \tau)$ with $<\tau>=(234 \pm 1) \mathrm{s}$

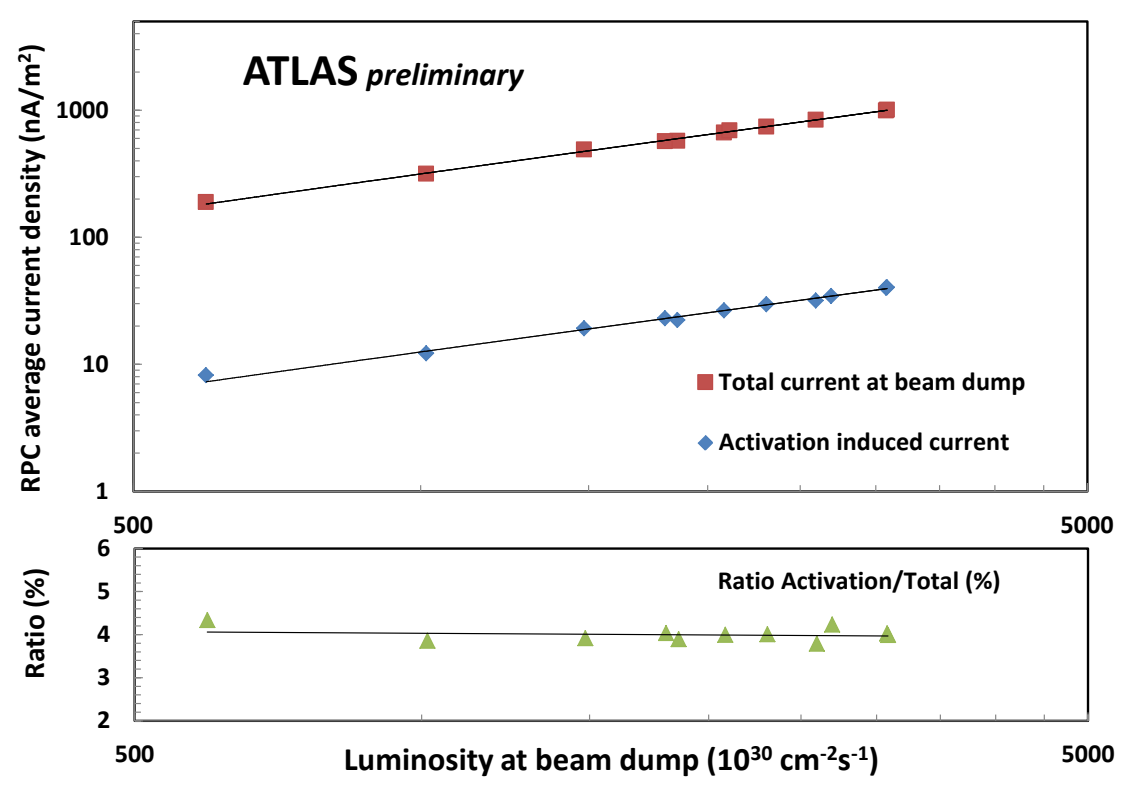

Figure 4: RPC Total average current, activation induced current (top) and their ratio activation/total current (bottom) as a function of the instantaneous luminosity at beam dump.

shown in Figure 2. Before providing an independent luminosity measurement some control checks were necessary.

As a first step, it was decided to monitor through the DCS the online ratio:

$$
R(t)=\frac{L_{A T L A S}(t)}{\Delta I_{R P C}(t)}
$$

in order to see if any systematic deviation was present during the LHC fill. No significant effects were observed. A further systematic check was the extraction of the ratio $R(t)$ at the beam dump for several fills where the conditions of the RPCs detector or the LHC bunch configuration were different. Some examples of modified conditions are listed here:

- before and after the LHC technical stops, when major interventions on the detector occurred; 
- a different gap selection (used by the averaging algorithm);

- a different LHC bunch configuration.

Figure 5 shows a quantitative comparisons between the reference ratio $R\left(t_{0}\right)$, extracted from a calibration run (at beam dump) and the ratios calculated for several fills with different number of colliding bunches during the 2011 data taking. It can be noticed that a maximum deviation of $\pm 1.5 \%$ was achieved; for the other systematic checks, similar or smaller fluctuations have been observed. The agreement and the stability of the result can be considered satisfactory.

In order to quote the RPCs luminosity for 2012 , a new reference ratio $R\left(t_{1}\right)$ will be used to calibrate the current with the ATLAS online luminosity measurement. This is mainly due to the changes on ATLAS shielding and LHC beam energies respect to 2011. Once this ratio is fixed, it will be possible to provide an independent measurement based on the following formula:

$$
L_{R P C}=R\left(t_{1}\right) \cdot \Delta I_{R P C}
$$

During the whole 2012, RPCs will be providing ATLAS with an independent luminosity measurement that will be used to monitor and understand potential discrepancies. The fact that the activation current was found to scale linearly with the luminosity, means that it will not be necessary to add a further systematic to the measurement.

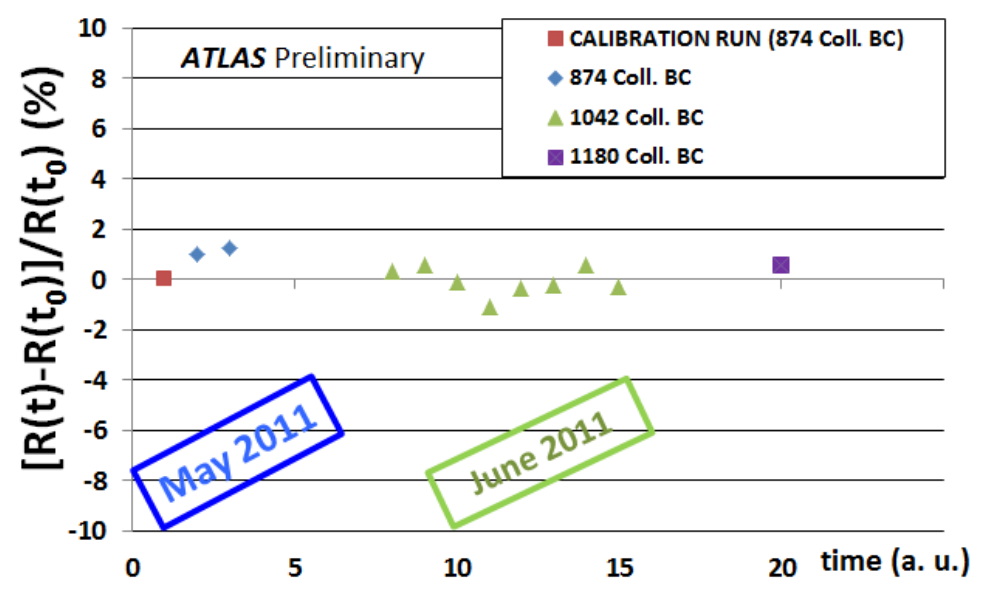

Figure 5: Percentage differences between the ratio $R(t)$ and the reference $R\left(t_{0}\right)$ (red square) calculated at beam dump for fills with a different number of colliding bunches.

\section{Conclusions}

The ATLAS RPCs proved to be very stable detector during the 2010 and 2011 data taking. The DCS monitoring capabilities made it possible to quantify the linear correlation between the LHC luminosity and the RPCs average current over four order of magnitude. A precise estimation of the cavern activation was extracted and found to be constant as a function of the luminosity. 
It has been shown how the control of the main systematics effects (environmental parameter correction, cavern activation, gap selection) is crucial to provide ATLAS with a stable and reliable luminosity measurement. The encouraging results obtained will be verified during 2012 data taking, where RPCs will be measuring the online luminosity together with the other ATLAS measurements.

\section{References}

[1] R. Santonico R. Cardarelli: "Development of resistive plate counters", NIM A, 187 (1981) 377.

[2] ATLAS Collaboration, "The ATLAS Experiment at the CERN Large Hadron Collider", JINST 3 (2008) S02003.

[3] ATLAS Muon Collaboration, "ATLAS Muon Spectrometer Technical Design Report", CERN/LHCC/97-22, (1997).

[4] A.Polini, "Performance and Operation of the ATLAS Resistive Plate Chambers Detector in 2011", RPC 2012, Frascati 2012, these proceedings.

[5] G. Chiodini, "The ATLAS RPC time-of-flight performance", RPC 2012, Frascati 2012, these proceedings.

[6] A. Polini, "Design and Performance of the Detector Control System of the ATLAS Resistive-Plate-Chamber Muon Spectrometer", J. Phys., Conf. Ser. 331022024.

[7] G. Aielli, "Cavern background measurement with the ATLAS RPC system", RPC 2012, Frascati 2012 , these proceedings. 